\title{
QUELLE CARPE DEVONS-NOUS PRÉSENTER SUR LE MARCHÉ ?
}

\author{
Par M. Raoul de la GIRAUDIÉRE \\ Président du Syndicat des propriètaires d'étıngs de Sologne.
}

Parmi les poissons cultivés dans nos étangs, les dévorants, Anguilles et Brochets, sont incontestablement les meilleurs, tant au point de vue de la qualité de la chair, que du prix de vente ; mais ils réclament pour leur alimentation une quantilé de poissons importante. Consommant plus qu'ils ne produisent, ils ne doivent être qu'un accessoire dans la production, où la Carpe occupe sans conteste le premier rôle.

La Carpe est un excellent poisson, à la condition dè la consommer grosse et bien en chair. Ce sont là les desiderata du marché ; d'où, faisant abstraction de nos anciennes mélhodes, nécessité d'améliorer notre pisciculture. Il était dans les habitudes de vendre la Carpe marchande à 500 grammes, voire même ì 375 grammes, ou tout venant, alevins non compris. Sage mesure, qu'il ne faut pas trop critiquer, parce que trop souvent, pour des raisons qui nous échappent, les alevins ne prennent pas leur accroissement normal. Mais cette manière de faire ne permet pas ì l'acheteur, toujours en présence d'un aléa, de donner les prix réservés aux poissons de belle qualité.

Le reproche fait à la Carpe d'être un nid à arêtes est fondé, si elle est de petite taille et de race abâtardie. De plus en plus le marché s'en éloigne, réservant ses faveurs à la Carpe d'un lilogramnie et plus.

Les conditions climatériques jouent un grand rôle, mais il n'est pas en notre pouvoir de les modifier à notre gré. Il en est d'autres, non moins importantes, qu'il nous appartient d'utiliser ou d'améliorer.

Trop souvent nous employons à l'empoissonnement des étangs des alevins d'origine inconnue, venus fortuitement de Carpes de qualité médiocre, ou de sujets trop jeunes.

Fréquemment, à la remise en eau d'un étang cultivé pendant plusieurs années, les alevins qui y sont immergés forcent, suivant l'expression consacrée, c'est-à-dire se mettent à frayer, donnant des alevins en grosse quantité. Ces alevins ne doivent être utilisés qu'à défaut d'autres. Un agriculteur n'élève pas, si beau soit-il, le produit d'une vache à son premier vélage. Prenons exemple sur lui, et n'utilisons que des alevins provenant de Carpes de choix, de deux à trois kilogrammes. La sélection des géniteurs se fait en prenant les sujets les mieux conformés : réduction au minimum des parties non comestibles : tête, squelelte, arêtes. Les Carpes doivent être courtes, de croissance rapide, avec les reins bien chamus. Jusqu'à ce qu'on soit arrivé, par une sélection méthodique, qui cst en cours, à redonner à la Carpe commune ces qualités qui lui font généra- 
lement défaul, prendre les céniteurs parmi les Carpes de races déjà sélectionnées : Carpes cuir, Carpes miroir, Carpes à écailles. Bien qu'elles soient toutes trois de précocité égale, mes préférences vont à ces dernières.

La richesse du fond et la qualité de l'eau des étangs sont variables, comme la nature des terrains. Nous dẹons régler l'ensemencement en alevins d'après l'expérience et la connaissance que nouṣ en avons. L'épandage de chaux et d'engrais après la pèche, le faucardage d'été qui, avec les bateaux actuellement construits, est de pratique facile, permettraient une immersion d'alevins plus nombreuse ; mais n'oublions pas que, pour un étang donné, les conditions climalériques restant les mèmes, le rendement en poids est constant, quel que scit le nombre de poissons immergés. Par conséquent, pour avoir des poissons plus gros, mettons un empoissonnement moins nombreux. Dans la pratique, pour la plupart de nos étangs de Sologne, ne pas mettre plus de $\mathrm{s} \delta \mathrm{a}$ à 200 alevins de deux étés à I'hectare. Nous obtiendrons ainsi l'accroissement voulu, surtout s'il s'agit d'alevins de races sélectionnées, et nous présenterons aux amateurs le beau et bon poisson qu'ils demandent.

\title{
CAUSES ESSENTIELLES DE DÉBOIRES ET DE SUCCÉS EN CARPICULTURE
}

\author{
Par M. Michel LHÉRITIER
}

Ex-Professeur interdépartemental d'aquiculture.

L'expérience d'autrui, n'a, dit-on jamais servi à personne. J'apporte, néanmoins, ma collaboration à la rédaction d'ume publication s'adressant spécialement à des gens de métier, car je les crois plus désireux d’idées pratiques que de littérature, capables de vérifier l'exactitude de mes allégations et de profiter d'une méthode s'inspirant des lois naturelles qui m'a réussi dans les milieux les moins propices à la production, à l'éducation et la sélection des Carpes.

J'y ai aussi été incité par la conviction que, nonobstant la propagaude menée, depuis rgio, par la Compagnie des chemins de fer de Paris a Orléans, sous la direction de son distingué ingénieur des Services commerciaux, M. Potwr ; les deux Congrès de l'étang et de l'élevage de la Carpe en r9r 8 et r924; les expositions piscicoles de Paris, Lyon, Limorres, etc., enfin les encouragements généreusement accordés par l'Etat, l'aquiculture nationale n'a pas progressé comme l'exigeait la situation économique.

Oui, quelques succès remarquables ont été enregistrés de ci, de là, par 